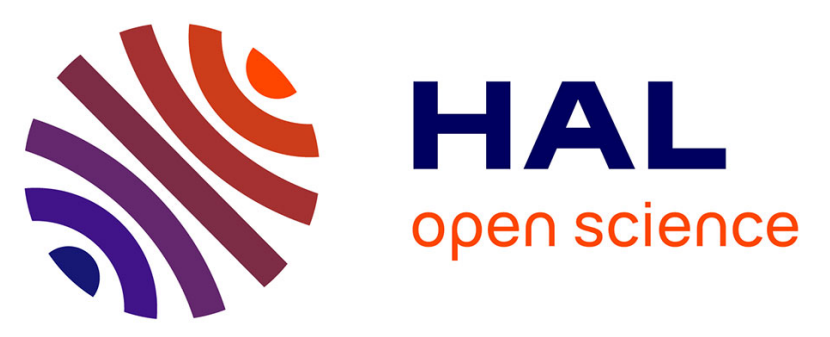

\title{
Detailed characterisation of focused ion beam induced lateral damage on silicon carbide samples by electrical scanning probe microscopy and transmission electron microscopy
}

\author{
F. Stumpf, A. A. Abu Quba, P. Singer, M. Rumler, Nikolay Cherkashin, \\ Sylvie Schamm-Chardon, Robin Cours, M. Rommel
}

\section{To cite this version:}

F. Stumpf, A. A. Abu Quba, P. Singer, M. Rumler, Nikolay Cherkashin, et al.. Detailed characterisation of focused ion beam induced lateral damage on silicon carbide samples by electrical scanning probe microscopy and transmission electron microscopy. Journal of Applied Physics, 2018, 123 (12), pp.125104. 10.1063/1.5022558 . hal-01745004

\author{
HAL Id: hal-01745004 \\ https://hal.science/hal-01745004
}

Submitted on 16 Apr 2018

HAL is a multi-disciplinary open access archive for the deposit and dissemination of scientific research documents, whether they are published or not. The documents may come from teaching and research institutions in France or abroad, or from public or private research centers.
L'archive ouverte pluridisciplinaire HAL, est destinée au dépôt et à la diffusion de documents scientifiques de niveau recherche, publiés ou non, émanant des établissements d'enseignement et de recherche français ou étrangers, des laboratoires publics ou privés. 


\section{Detailed characterisation of focused ion beam induced lateral damage on silicon carbide samples by electrical scanning probe microscopy and transmission electron microscopy}

F. Stumpf, A. A. Abu Quba, P. Singer, M. Rumler, N. Cherkashin, S. Schamm-Chardon, R. Cours, and M. Rommel

Citation: Journal of Applied Physics 123, 125104 (2018); doi: 10.1063/1.5022558

View online: https://doi.org/10.1063/1.5022558

View Table of Contents: http://aip.scitation.org/toc/jap/123/12

Published by the American Institute of Physics

\section{Articles you may be interested in}

Optical properties of InGaN thin films in the entire composition range

Journal of Applied Physics 123, 125101 (2018); 10.1063/1.5020988

Fabrication of phonon-based metamaterial structures using focused ion beam patterning Applied Physics Letters 112, 091101 (2018); 10.1063/1.5008507

Piezoelectric field, exciton lifetime, and cathodoluminescence intensity at threading dislocations in $\mathrm{GaN}\{0001\}$ Applied Physics Letters 112, 122101 (2018); 10.1063/1.5022170

Characteristics of GaN-based $500 \mathrm{~nm}$ light-emitting diodes with embedded hemispherical air-cavity structure Journal of Applied Physics 123, 125702 (2018); 10.1063/1.5018340

Thermal conductivity of ternary III-V semiconductor alloys: The role of mass difference and long-range order Journal of Applied Physics 123, 125103 (2018); 10.1063/1.5008262

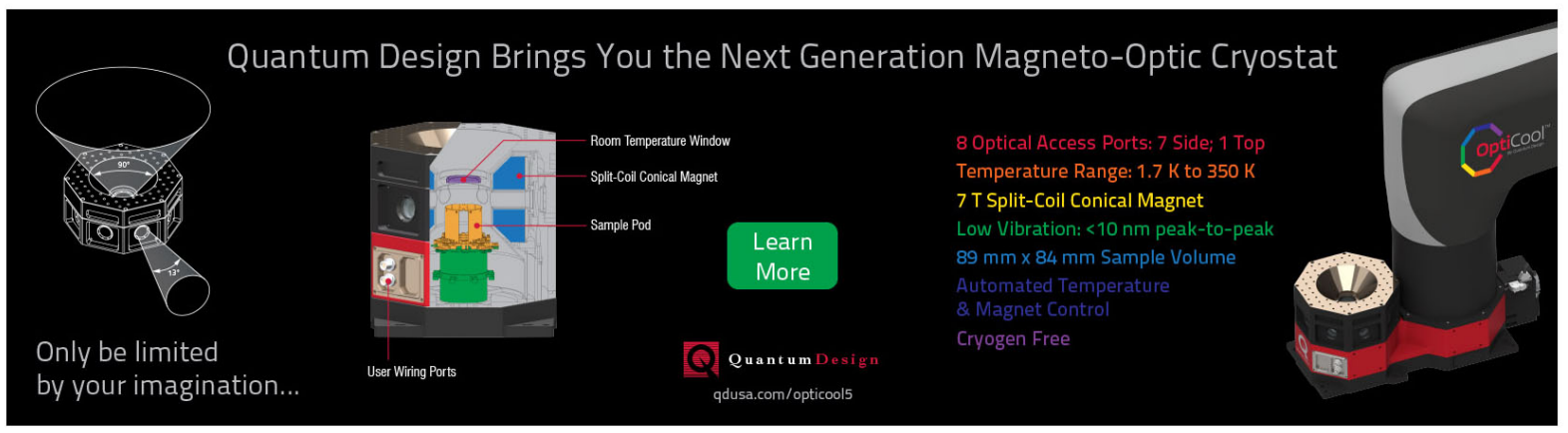




\title{
Detailed characterisation of focused ion beam induced lateral damage on silicon carbide samples by electrical scanning probe microscopy and transmission electron microscopy
}

\author{
F. Stumpf ${ }^{1,2}$ A. A. Abu Quba, ${ }^{1}$ P. Singer ${ }^{3}$ M. Rumler, ${ }^{2,3}$ N. Cherkashin, ${ }^{4}$ \\ S. Schamm-Chardon, ${ }^{4}$ R. Cours, ${ }^{4}$ and M. Rommel ${ }^{1}$ \\ ${ }^{1}$ Fraunhofer Institute for Integrated Systems and Device Technology (IISB), Erlangen 91058, Germany \\ ${ }^{2}$ Erlangen Graduate School in Advanced Optical Technologies (SAOT), Erlangen 91052, Germany \\ ${ }^{3}$ Chair of Electron Devices (LEB), University of Erlangen-Nuremberg, Erlangen 91058, Germany \\ ${ }^{4}$ CEMES, Université de Toulouse, CNRS, 29 Rue Jeanne Marvig, Toulouse 31055, France
}

(Received 16 January 2018; accepted 7 March 2018; published online 27 March 2018)

\begin{abstract}
The lateral damage induced by focused ion beam on silicon carbide was characterized using electrical scanning probe microscopy (SPM), namely, scanning spreading resistance microscopy and conductive atomic force microscopy (c-AFM). It is shown that the damage exceeds the purposely irradiated circles with a radius of $0.5 \mu \mathrm{m}$ by several micrometres, up to $8 \mu \mathrm{m}$ for the maximum applied ion dose of $10^{18} \mathrm{~cm}^{-2}$. Obtained SPM results are critically compared with earlier findings on silicon. For doses above the amorphization threshold, in both cases, three different areas can be distinguished. The purposely irradiated area exhibits resistances smaller than the non-affected substrate. A second region with strongly increasing resistance and a maximum saturation value surrounds it. The third region shows the transition from maximum resistance to the base resistance of the unaffected substrate. It correlates to the transition from amorphized to defectrich to pristine crystalline substrate. Additionally, conventional transmission electron microscopy (TEM) and annular dark-field STEM were used to complement and explain the SPM results and get a further understanding of the defect spreading underneath the surface. Those measurements also show three different regions that correlate well with the regions observed from electrical SPM. TEM results further allow to explain observed differences in the electrical results for silicon and silicon carbide which are most prominent for ion doses above $3 \times 10^{16} \mathrm{~cm}^{-2}$. Furthermore, the conventional approach to perform current-voltage measurements by c-AFM was critically reviewed and several improvements for measurement and analysis process were suggested that result in more reliable and impactful c-AFM data. Published by AIP Publishing.
\end{abstract}

https://doi.org/10.1063/1.5022558

\section{INTRODUCTION}

Today's modern electronics are heading toward the miniaturization of components and devices down to the nanometre scale. This miniaturization process requires technologies and tools that support reliable results in manufacturing and characterization. In this development of downscaling, focused ion beam (FIB) has established itself as a flexible tool for prototyping, ${ }^{1}$ direct patterning, ${ }^{2}$ cross-section preparation and analysis or transmission electron microscope (TEM) lamella preparation, ${ }^{3}$ as the beam can be focused onto spot sizes of the nanometre scale (nominal diameter $<6 \mathrm{~nm}$ ). ${ }^{4}$ However, it has already been shown that the Gaussian beam shape of the FIB causes damage outside the purposely irradiated area on silicon. ${ }^{5-9}$ The lateral damage might spread out over several micrometres from the purposely irradiated area and thus the localized character of FIB processing has to be re-evaluated. Several methods have been applied to study the lateral damage extension, e.g., atomic force microscopy (AFM) topography measurements, ${ }^{10}$ TEM analyses, ${ }^{11,12}$ carrier mobility measurements, ${ }^{13}$ and scanning capacitance microscopy. ${ }^{14}$ For silicon substrates, consistent results have been achieved by scanning spreading resistance measurements (SSRM) in our earlier work. ${ }^{5,15}$
Recently, in the field of power electronics, the performance of $\mathrm{Si}$ devices has almost reached theoretical limits. Further improvements require investigation of new materials. Due to their advantageous properties, wide-bandgap materials, such as Al- and Ga-based semiconductors or other compound semiconductors like silicon carbide ( $\mathrm{SiC}$ ), are promising alternatives. ${ }^{16,17}$ Thus, the demand for the characterization of such a material will significantly increase, including in-depth analyses using FIB. Due to the still rather high costs of $\mathrm{SiC}$ substrates, product wafers might have to be analysed during development. Therefore, it is of great interest to understand the amount and extent of damage created during the FIB investigation.

To specifically estimate the lateral extent of FIB induced damage, SSRM and TEM characterization of FIB irradiated $4 \mathrm{H}-\mathrm{SiC}$ substrate samples were performed. To allow a direct comparison of the results, the approach was similar to the one we already applied for the silicon case. ${ }^{5}$ Additionally, conductive atomic force microscopy (c-AFM) was applied to provide additional and partly complementary information on the influence of the electrically relevant defects on local current-voltage characteristics. Moreover, possible improvements to c-AFM measurement and analysis in general are suggested. 


\section{EXPERIMENTAL SET-UP AND SAMPLE PREPARATION}

Before FIB irradiation, all silicon carbide substrate samples $(4 \mathrm{H}-\mathrm{SiC}$, highly nitrogen $\mathrm{n}$-doped with a doping concentration in the range of $0.8 \times 10^{19} \mathrm{~cm}^{-3}-2.0 \times 10^{19} \mathrm{~cm}^{-3}$ ) were cleaned thoroughly to prevent surface contamination affecting the results. The cleaning procedure consisted of a ten-minute acetone ultrasonic bath, followed by a ten-minute isopropyl ultrasonic bath, a five-minute DI water ultrasonic bath, and a $\mathrm{HF}$ dip to remove the native oxide ( $15 \mathrm{~s}$ at $5 \% \mathrm{HF})$. In order to provide a direct comparison with our former results on silicon, the identical FIB irradiation patterns were chosen. ${ }^{5}$ Consequently, twelve circular spots on the silicon carbide samples were irradiated with different ion doses. The applied ion doses were varied from $3.0 \times 10^{12} \mathrm{~cm}^{-2}$ to $1.0 \times 10^{18} \mathrm{~cm}^{-2}$ with the pattern diameter fixed at $1 \mu \mathrm{m}$, the ion current at $1.5 \mathrm{pA}$, and the beam energy at $30 \mathrm{keV}$. The chamber pressure was kept at $1-2 \times 10^{-6}$ mbar to reduce the interaction between the $\mathrm{Ga}^{+}$ions and residual gas particles in the vacuum, as these interactions were found to be the main reason for FIB beam broadening. ${ }^{5}$ This pressure equals the pressure after pumping for an entire night and equals the lowest possible pressure in the used FIB set-up. All samples were irradiated with a FEI Helios Nanolab 600 Focused Ion Beam system equipped with $\mathrm{a} \mathrm{Ga}^{+}$liquid metal ion source. It is important to note that all irradiations were done without imaging the irradiated area, as this would have impacted the samples additionally.

To achieve optimal set-up conditions for electrical scanning probe microscopy (SPM) measurements, the samples were moved to the AFM immediately after FIB processing, in order to minimize the growth of a native oxide. All SSRM and c-AFM measurements were performed with a Bruker Dimension ICON system equipped with a Nanoscope V Controller and the SSRM and TUNA2 application modules, respectively. For both methods, standard boron doped diamond coated Si AFM probes, with a nominal tip radius of $35 \mathrm{~nm}$, showed the most reproducible results. In this work explicitly, CDT-FMR tips (resonance frequency $\mathrm{f}_{0}=105 \mathrm{kHz}$, spring constant $\mathrm{C}=6.2 \mathrm{~N} / \mathrm{m}$ ) from Nanoworld AG were used for electrical measurements. High-resolution topography measurements, i.e., tapping mode measurements, were performed to verify the topography obtained during the electrical measurements and, thus, to validate the results. Those tapping mode measurements were performed after the electrical analysis, as the native oxide does not significantly alter the tapping mode measurements. For those measurements, standard Si AFM probes with a nominal tip radius of less than $10 \mathrm{~nm}$ were used $(\mathrm{NCH}$ tips from Nanoworld $\mathrm{AG}, \mathrm{f}_{0}=320 \mathrm{kHz}$, and $\mathrm{C}=42 \mathrm{~N} / \mathrm{m}$ ). A detailed discussion of the two electrical SPM methods can be found in the literature. ${ }^{18-21}$ In particular, Binning et al. describe the AFM in general ${ }^{22}$ and Zhong et al. the tapping mode. ${ }^{23}$

The following SSRM parameters were determined to give reproducible results during this work and were always used, if not stated otherwise. The DC bias voltage was set to $-3 \mathrm{~V}$ and was applied to the sample, while the tip was kept grounded. This voltage leads to reasonable resistance measurements and prevents anodic oxidation of the sample (see Fig. 1 and the next paragraph). The other important parameter

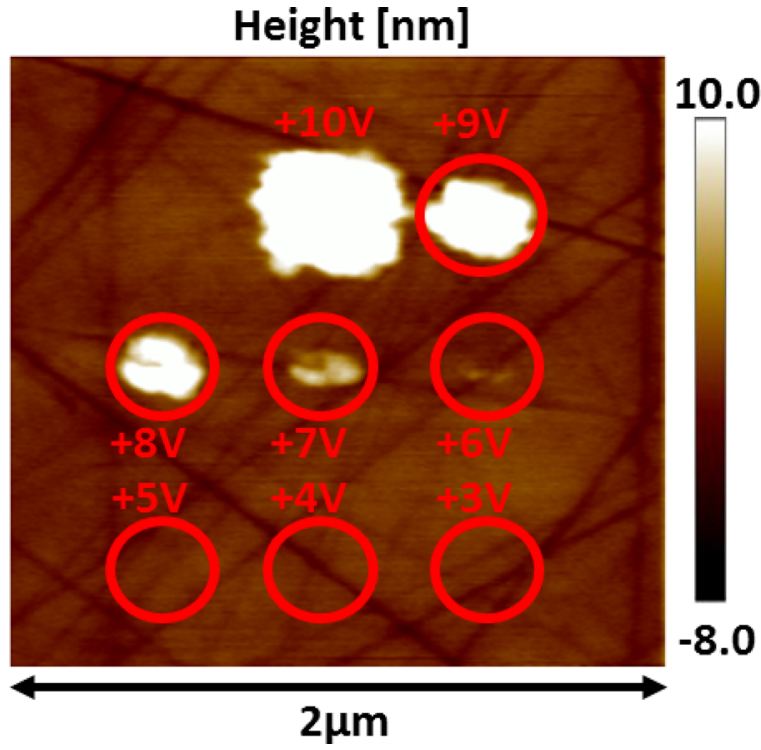

FIG. 1. Tapping mode measurement of a non-irradiated SiC sample after performing different $\mathrm{I}-\mathrm{V}$ ramps from $0 \mathrm{~V}$ to a maximum voltage (i.e., $3 \mathrm{~V}$ to $10 \mathrm{~V}$ ) as indicated in the figure. The red circles help guide the eye to the dedicated spots of the applied ramp (except for the highest voltage).

of SSRM, the applied force between tip and sample, was set to $4.0 \mu \mathrm{N}$ for each measurement. This force resulted in a reliable electrical contact between tip and sample while causing no visible damage on the surface. The amplifier used in the SSRM module is logarithmic and thus the measured resistance is on a logarithmic scale. Accordingly, the unit of all SSRM measurements will be written as $\log (\mathrm{Ohm})$ to display this fact, i.e., $8 \log (\mathrm{Ohm})$ equals $10^{8} \Omega$.

For c-AFM measurements, the same force was used as for SSRM, to ensure the same electrical contact and good comparison between the two methods. While for SSRM the voltage was set to a fixed value and the sample was mapped in one measurement, for c-AFM, the voltage was ramped in a certain interval on specific points on the sample. The voltage interval was chosen from $-5 \mathrm{~V}$ to $5 \mathrm{~V}$, to not only cover the same voltage as in the SSRM measurements, but also to observe the sample behaviour as a function of other voltage regimes. The maxima of the ramps were limited to minimize the influence of anodic oxidation of the sample. To determine the voltage threshold of significant anodic oxidation, different voltage ramps were applied with an increasing maximum voltage from $3 \mathrm{~V}$ up to $10 \mathrm{~V}$. The results that were measured with tapping mode afterwards are shown in Fig. 1, where the red circles help indicate the positions of the applied ramps. As there is no oxidation observable for voltages below $6 \mathrm{~V}$, all further measurements were limited to $\pm 5 \mathrm{~V}$.

Complementary information to support the interpretation of the electrical behaviour of the samples was obtained using conventional TEM imaging (Jeol 2010 operating at $200 \mathrm{kV}$ ) and annular dark-field/electron energy loss spectroscopy in the scanning transmission electron microscopy mode (ADFSTEM and EELS-STEM; FEI Tecnai ${ }^{\text {TM }}$ F20 operating at $200 \mathrm{kV}$ ) on identically prepared FIB irradiated samples. The TEM lamellae were thinned down to thicknesses of $100 \mathrm{~nm}$ with the FEI Helios Nanolab 600 FIB, followed by low $5 \mathrm{kV}$ voltage ion polishing of the lamellae. 


\section{IMPROVEMENT OF c-AFM MEASUREMENTS AND ANALYSIS}

For a better understanding of the results, it is important to know that c-AFM can be considered as a statistical method in the sense that I-V curves on the same samples are rarely completely identical and single outliers could happen and affect the results quite significantly. ${ }^{24-26}$ Thus, c-AFM results are usually averaged measurements of several repetitions. The standard approach that is most commonly used simply takes the average of all currents for each voltage value. This method will be labelled as "I-average" further on. Another approach, designed in this work, is interpolating each individual curve linearly and then calculating the mean voltage for each current value, labelled as "V-average." For a better understanding, explanation and visualization of the effect on the final results, a typical example is illustrated in Fig. 2. Two c-AFM ramps taken on the same sample are shown split into negative [Fig. 2(a)] and positive [Fig. 2(b)] voltage regimes. For the negative voltage regime, it is apparent that as soon as the first ramp hits the current limit of the set-up, in this case $12 \mathrm{nA}$, the
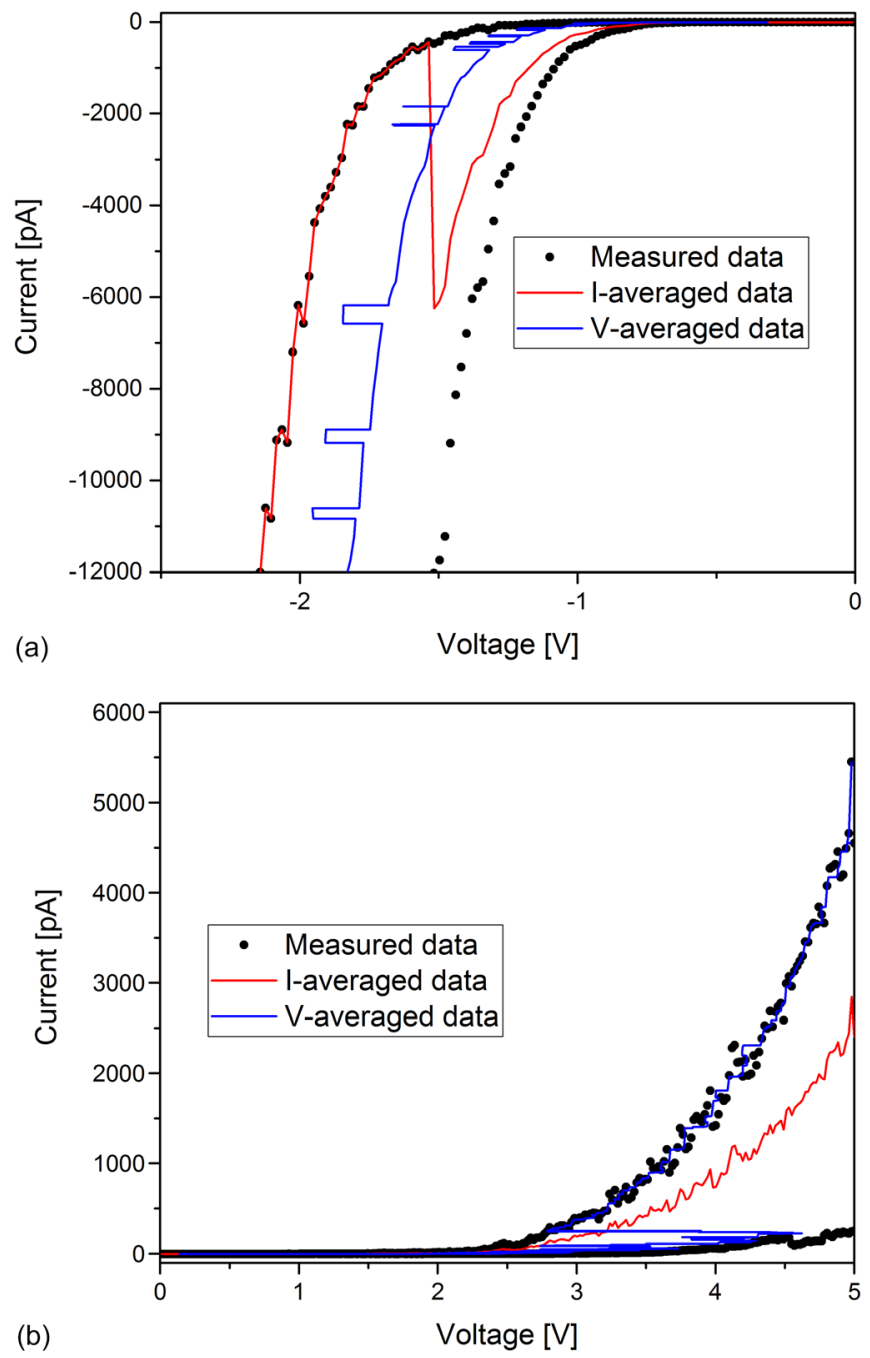

FIG. 2. Comparison of voltage- and current-averaged analysis for different voltage intervals, (a) negative voltages and (b) positive voltages. The black dots represent the measured values for two different c-AFM ramps on the same sample. The red and blue graphs show the two different approaches of data averaging, I-averaging and V-averaging, respectively.
I-averaged curve (red curve) represents the curve that does not reach the limit yet (note the sudden jump at around $-1.5 \mathrm{~V}$ ). This is caused by the AFM software that does not record currents that reach the compliance value and, thus, excludes them from the analysis. An improvement could be achieved by manually setting the values to the compliance current instead of discarding them and, thus, offsetting the average. This average would still not represent an appropriate average as the currents were artificially limited. This issue is solved by our approach of $\mathrm{V}$-averaging as all curves in this regime cover the entire current spectrum and, thus, are included in the averaging [blue curve in Fig. 2(a)].

In the positive voltage regime, the observation follows the exact opposite [see Fig. 2(b)]. The I-averaged curve (red curve) properly represents the average of the measured data and the $\mathrm{V}$-averaged curve (blue curve) aligns with the upper data points once the current is above the maximum current of the lower set of data points. The standard I-averaging works well as the curves include all current values for each available voltage. Thus, averaging at each voltage will include every data point of each curve. The $\mathrm{V}$-averaging, however, aligns with the upper c-AFM curve, as the lower curve does not share all the same current values and, thus, is discarded for the averaging process at these currents. Concluding from these observations, all further measurements will be averaged according to the specific voltage regime of the measurement. Those observations are not only applicable to this work, but also apply to c-AFM measurements in general. The different averaging methods are needed because the currents behave differently for either the forward or the reverse direction of the Schottky contact. ${ }^{27}$ In the case of a p-doped substrate material, both averaging methods should be used for the opposite voltage regimes.

Regarding the measurement set-up, c-AFM curves are usually performed as single ramps from a starting voltage to an end voltage, e.g., $-5 \mathrm{~V}$ to $5 \mathrm{~V}$ and automatically back to the starting voltage. This standard approach will be labelled "single ramp" further on. Another approach, designed in this work is splitting the ramps into two separate parts during the measurement and combining them mathematically afterwards, labelled "combined ramp." In this work, the common and starting point for all combined ramps was set to $0 \mathrm{~V}$ and the end voltage of the separate parts was either $-5 \mathrm{~V}$ or $5 \mathrm{~V}$. The voltage steps per ramp were chosen to be 512 for single ramps and 256 for each of the two individual ramps of the combined ramps. For comparison of the two approaches, cAFM measurements were performed on a non-irradiated $\mathrm{SiC}$ sample. In Fig. 3, each curve is determined by either 25 consecutive measurements on a single spot "Point $(1 \times 25)$ " or by 25 individual measurements arranged in a $5 \times 5$ grid on the sample "Matrix $(5 \times 5)$ ". First of all, the same expected Schottky behaviour of the set-up as shown in Fig. 2, caused by a metallic tip on a semiconductor surface, is measurable and ensures the validity of the measurements.

Secondly, it is apparent that for the point ramps (grey and orange curves) in the negative voltage regime, the onset of significant current occurs for smaller voltages (considering absolute values) compared with the corresponding matrix ramps (black and red curves) due to the repeated measurements on 


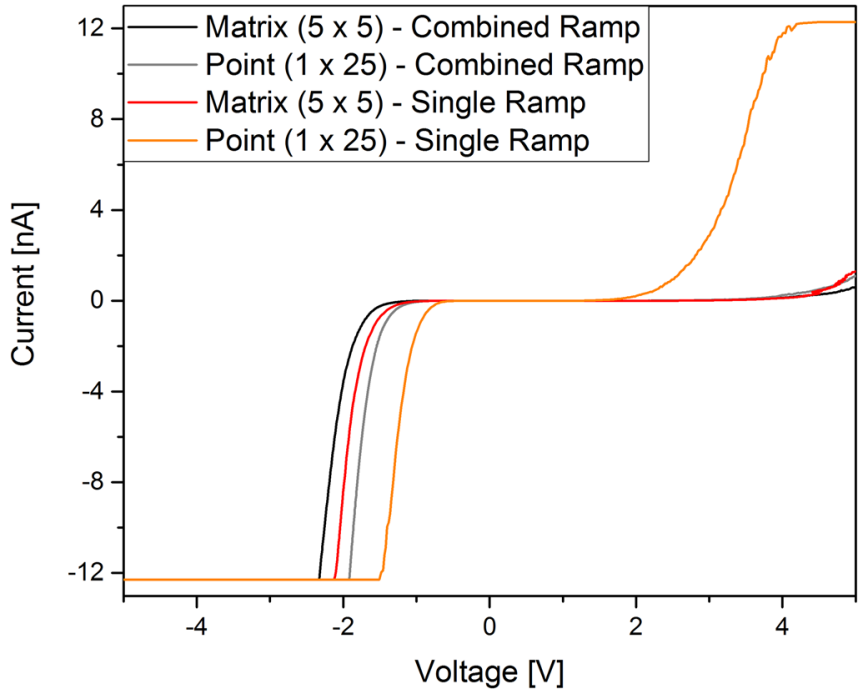

FIG. 3. Comparison of different I-V curve measurement approaches, "matrix" versus "point" and "combined ramp" versus "single ramp". "Matrix" defines the average curve of the measurements in a $5 \times 5$ array in contrast to the 25 measurements in a single "point." For "single ramp," voltage ramps were applied in one ramp from $-5 \mathrm{~V}$ to $5 \mathrm{~V}$. The "combined ramp" is a combination of two split measurements from $0 \mathrm{~V}$ to $\pm 5 \mathrm{~V}$.

the same spot. This effect is much more pronounced for the "Single Ramp" measurement than for the "Combined Ramp" measurement. In addition, the "Point $(1 \times 25)$ - Single Ramp" exhibits rather high currents for positive voltages, whereas all other ramps do not. This is caused by the high voltage $(-5 \mathrm{~V})$ that is instantly applied to the material at the start of each "Point $(1 \times 25)$ - Single Ramp" during the 25 ramp cycle. This affects all further measurements on this spot and, thus, makes further repetitions neither reliable nor reproducible. This effect might also be observable for the "Matrix $(5 \times 5)$ - Single Ramp" which already shows a slightly higher current increase for the maximum positive voltages compared with the combined ramps, as the changes to the sample already affect the measurements within a single measurement. For the combined ramps, on the other hand, the voltages are steadily increased, starting from $0 \mathrm{~V}$ and then reversed back to $0 \mathrm{~V}$. Thus, there is no abrupt change in the electrical field and the impact on the sample is drastically reduced and the results are more reproducible. Therefore, all further measurements in this work will be combined ramps to get the most reliable results and statistical outliers can be easily discarded. Furthermore, the focus will be on measurements in the forward direction of the Schottky contact, so all further ramps will be from $0 \mathrm{~V}$ to $-5 \mathrm{~V}$.

\section{RESULTS AND DISCUSSION}

First, SSRM results will be shown comparing FIB induced electrically detectable damage and the topography of the samples as a function of the applied ion dose. Furthermore, similarities and differences between the results for the different materials $\mathrm{Si}$ and $\mathrm{SiC}$ will be discussed considering the results of our former work on $\mathrm{Si}^{5}$ (see following Sec. IV A). Based on TEM results, a possible explanation for the observed electrical effects will be given. The results from Sec. IV A will be compared with the c-AFM measurements on the same structures on $\mathrm{SiC}$ in $\mathrm{Sec}$. IV B.

\section{A. Scanning spreading resistance microscopy}

The prepared $\mathrm{SiC}$ samples were measured with SSRM and an exemplary result is shown in Fig. 4. The scratches on the surfaces [Fig. 4(a)] are caused by the vendor's polishing of the original $\mathrm{SiC}$ wafers prior to the FIB sample preparation. As other samples with different scratch patterns showed the same results, the influence of the scratches on the SSRM measurements can be considered as negligible. For low irradiation doses (below $1 \times 10^{14} \mathrm{~cm}^{-2}$ ), no noticeable topography changes are measured at the irradiated areas. The amorphization threshold of $4 \mathrm{H}-\mathrm{SiC}$ substrate is approximately $1 \times 10^{14} \mathrm{~cm}^{-2}$ according to literature, comparable to the one of $6 \mathrm{H}-\mathrm{SiC}^{28,29}$ Above this threshold, the irradiated spots are clearly visible as bright areas in the topography map. This indicates a rise in the surface due to the substrate swelling caused by the $\mathrm{Ga}^{+}$ions amorphizing the silicon carbide. ${ }^{30}$ At the highest applied irradiation doses, crater structures due to FIB milling are observed (dark spots). The impact of the FIB irradiation on the electrical properties of the material is evidenced by the corresponding SSRM signal [Fig. 4(b)]. For dose values well below the amorphization threshold, the resistance within the irradiated area is higher (indicated by the brighter colour) compared with the resistance of the unaffected areas. This is most likely due to lattice defects caused by the $\mathrm{Ga}^{+}$ions which significantly lower carrier mobility.

For doses closer to the amorphization threshold and above, the observable electrical behaviour is similar to the results in our previous work on FIB irradiated Si samples. ${ }^{5}$ The purposely irradiated areas exhibit a decrease in resistance and are surrounded by areas with increased resistance. The increase in the conductivity after $\mathrm{Ga}^{+}$irradiation can be explained by the transition from defect-rich crystalline silicon carbide to amorphous silicon carbide, as suggested by Beuer et al. ${ }^{31}$ for silicon. In the outmost areas where the Ga dose is not sufficient for amorphization anymore, the resistance increases due to the impinging $\mathrm{Ga}^{+}$ions that cause crystal defects according to the observations for lower ion doses. The extent of this area increases with the irradiation dose, up to approximately $8 \mu \mathrm{m}$ for the highest dose.

Cross-sections of the topography and the electrical signal of the area irradiated with a dose of $3 \times 10^{17} \mathrm{~cm}^{-2}$ illustrate the observed changes in detail [Fig. 4(c)]. The topographical section (black line) shows the nominally expected $1 \mu \mathrm{m}$ width of the purposely irradiated area. The "w-shape" in the bottom of the irradiation hole is caused by enhanced sputtering due to ion scattering at the non-vertical sidewalls, a well-known effect of FIB irradiation at these doses. ${ }^{32}$ The cross-section of the spreading resistance signal [red curve in Fig. 4(c)] shows a minimum of around $8.0 \mathrm{log}(\mathrm{Ohm})$ within the purposely irradiated area. This is presumably explained by the high density of Ga nanoclusters within the amorphization layer that improve conductivity. They were imaged and analysed by TEM-EELS and will be discussed later. Thus, the further from the purposely irradiated area, the higher the resistance as the amorphization layer contains fewer $\mathrm{Ga}$ nanoclusters 


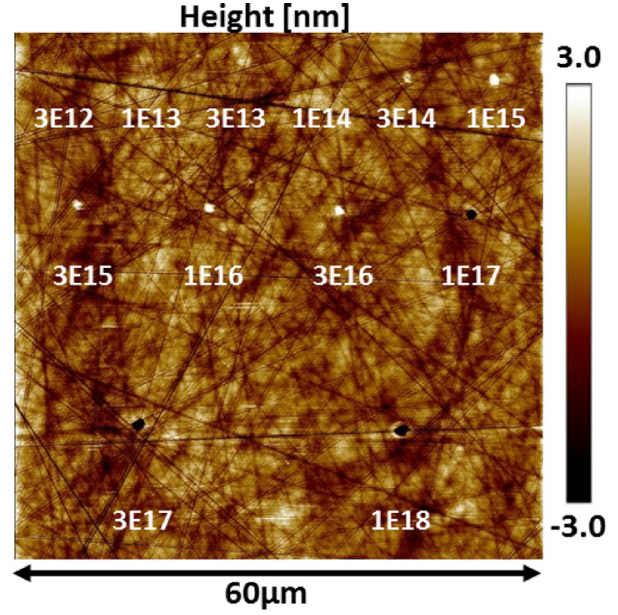

(a)

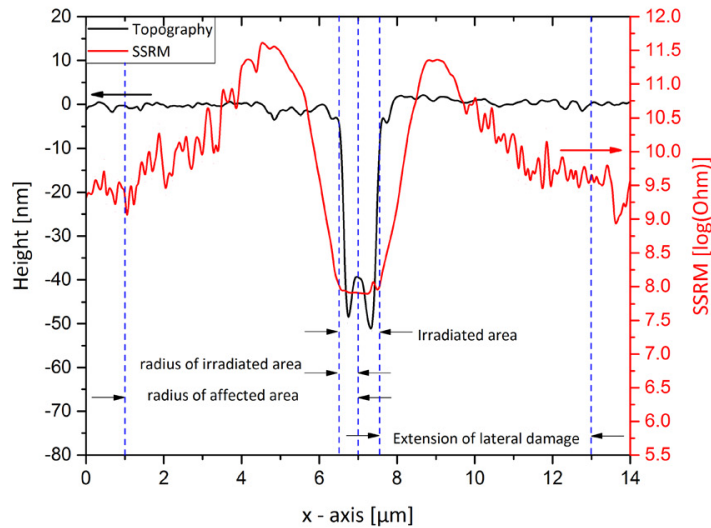

(c)

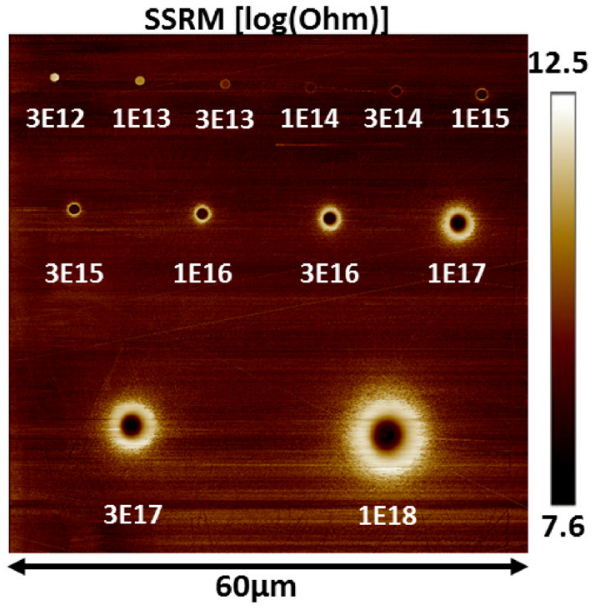

(b)

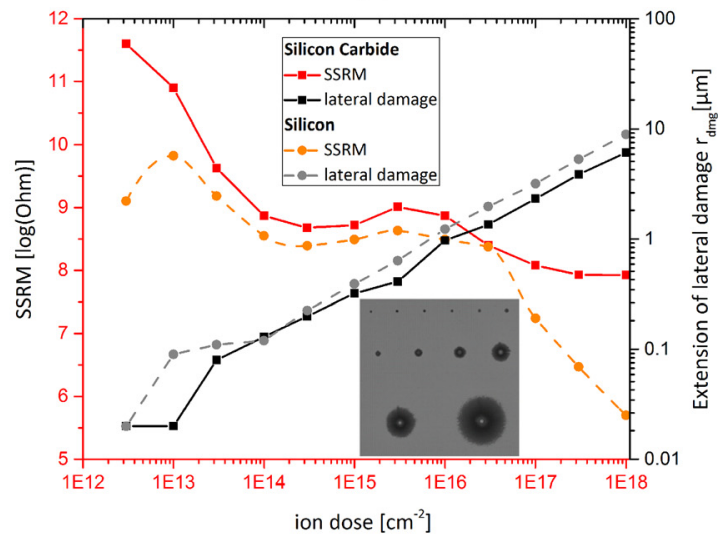

(d)

FIG. 4. (a) Topography of SiC sample with $1 \mu \mathrm{m}$ diameter circular structures irradiated by FIB with various doses $\left(3 \times 10^{12} \mathrm{~cm}^{-2}-1 \times 10^{18} \mathrm{~cm}^{-2}\right.$ as indicated in the figure). (b) Scanning spreading resistance measurement of the same sample. Brighter areas indicate an increase in resistance due to the FIB induced damage. (c) Sections of the structure irradiated with a dose of $3 \times 10^{17} \mathrm{~cm}^{-2}$ : topography (black line) and SSRM signal (red line). The blue dashed lines help guide the eye by visualizing the irradiated and affected areas. (d) Plot of lateral damage extension and SSRM signal of all doses for SiC (i.e., black and red graphs, squared symbols) and for Si (grey and orange dashed graphs, circular symbols; values taken from our previous work ${ }^{5}$ ). The connecting lines help guide the eye. The inset shows the SSRM measurements for Si, using an inverted colour scheme, i.e., darker areas equal higher spreading resistance [Reprinted with the permission from Rommel et al. J. Vac. Sci. Technol. B 28, 595 (2010). Copyright 2010, American Vacuum Society]. ${ }^{5}$

and atoms due to the decreasing amount of impinging $\mathrm{Ga}^{+}$ ions. This behaviour changes at the transition from amorphized to defect-rich crystalline SiC, where after reaching the peak resistance of $11.5 \mathrm{log}(\mathrm{Ohm})$, the amorphization threshold is passed and the impinging $\mathrm{Ga}^{+}$ions create crystallographic defects. Onwards, the lattice damage decreases and, thus, the resistance drops accordingly, observable as the speckled area in Fig. 4(b).

The affected area outside the purposely irradiated area is defined by the following radius $r_{\mathrm{dmg}}$ :

$$
r_{d m g}=r_{a f f}-r_{i r r},
$$

where the radius of the total affected area is $r_{\text {aff }}$ and the radius of the purposely irradiated area is $r_{\text {irr }}$. Determining the extension of the lateral damage for all irradiation doses leads to the diagram plotted in Fig. 4(d), with the grey (circular symbols) and black (squared symbols) graph representing $\mathrm{Si}^{5}$ and $\mathrm{SiC}$, respectively. It should be noted that the values for an irradiation with doses below $3 \times 10^{13} \mathrm{~cm}^{-2}$ may be discarded for quantitative evaluation, as the measurement uncertainty is quite substantial compared with the actual values of $r_{d m g}$. Fitting the remaining doses, the lateral damage extension shows a steady and linear increase in the double logarithmic plot for both materials. The calculation of the power law dependency results in

$$
\text { dose } \sim\left(r_{d m g}\right)^{x}\left\{\begin{array}{l}
x_{S i}=2.21 \\
x_{S i C}=2.45 .
\end{array}\right.
$$

The difference between both values is mainly due to the small radius value for $\mathrm{Si}$ at $1 \times 10^{14} \mathrm{~cm}^{-2}$, as this changes the slope of the graph accordingly. Apart from that point, the curves showing the lateral spread of the irradiation damage run almost parallel for the two materials with Si showing slightly higher absolute values.

This rather similar behaviour, in principle, was expected because the damage extension is mainly caused by the size of the focused ion beam which is obviously not dependent on the substrate material, and thus, the relative increase in the irradiated area with increasing dose should be equal for appropriate materials. Furthermore, both samples are highly 
doped so that the influence of the irradiation induced defects on the change of conductivity should be rather similar.

On the other hand, from the experimental point of view, electrical SPM on SiC is much more complicated due to the hardness of $\mathrm{SiC}$ and its large band gap. Both lead to high contact resistances between the SPM tip and the sample. Therefore, the obtained similarity in the experimentally obtained results proves that even on $4 \mathrm{H}-\mathrm{SiC}$, very reliable quantitative electrical SPM measurements are possible. Only the sensitivity of the measurements on $\mathrm{SiC}$ is decreased due to the higher contact resistance which in turn results in the slightly larger spread of the detectable damage for $\mathrm{Si}$. Thus, for $\mathrm{Si}$, even smaller defect densities can be detected and therefore a wider area is determined to be affected by the irradiation.

Comparing the SSRM signals within the irradiated areas [orange and red graphs in Fig. 4(d)] and discarding the results for the smallest dose, both materials show a similar behaviour until reaching doses above $3 \times 10^{16} \mathrm{~cm}^{-2}$. For the samples in this work, the base resistance of the SiC substrate $(\rho \approx 10-30 \mathrm{~m} \Omega \mathrm{cm})^{33}$ is slightly higher than that of the $\mathrm{Si}$ substrate $(\rho \approx 9.9 \mathrm{~m} \Omega \mathrm{cm})$ used in our previous work. ${ }^{5}$ This explains the slight offset in the resistance curves, whereas the shapes of the curves are comparable. Further on, the spreading resistance decreases, as more material is amorphized by the $\mathrm{Ga}^{+}$ions, until the dose reaches $1 \times 10^{15} \mathrm{~cm}^{-2}$. Then, a slight increase in the spreading resistance is measured, which could be caused by the increasing thickness of the amorphization layer. For doses above approximately $3 \times 10^{16} \mathrm{~cm}^{-2}$, the spreading resistance drops drastically for $\mathrm{Si}$, whereas for $\mathrm{SiC}$, the resistance only slightly decreases and seems to saturate for the highest doses.

To analyse the origin for this difference, two crosssectional specimens of the $\mathrm{SiC}$ substrates irradiated with $\mathrm{Ga}$ doses of $1 \times 10^{17} \mathrm{~cm}^{-2}$ and $1 \times 10^{18} \mathrm{~cm}^{-2}$ were investigated by TEM. Conventional Bright-field and Dark-field TEM imaging and ADF/EELS-STEM experiments have been applied for a detailed analysis (at a nanometric scale) of the structural and compositional properties of the superficial regions subjected to intentional and non-intentional irradiation.

The Bright-field image shown in Fig. 5(a) presents the overview of the structure irradiated with a $\mathrm{Ga}$ dose of $1 \times 10^{18} \mathrm{~cm}^{-2}$. Three zoomed parts of the different regions of the image are added to easily distinguish between the amorphous regions (presenting no diffraction contrast), damaged crystalline regions (presenting strong contrast variation), and "perfect" crystalline regions (presenting homogeneous diffraction contrast). According to the difference in the structural properties of the superficial layer, the image can be split into three different regions which are labelled as sections I, II, and III.

"Section I" contains a $\sim 1 \mu \mathrm{m}$ wide valley which corresponds to the purposely and, thus, homogeneously irradiated region of the substrate. The $45 \mathrm{~nm}$ thick all amorphous superficial layer in the valley contains a dense array of randomly distributed nanoparticles (see the dark spots in right-side inset), which are mostly concentrated within its upper part. The detailed analysis of their composition by EELS-STEM measurements revealed that they are composed of Ga [Fig. 5(b)]. The Ga nanoparticles are also contained within a $13 \mathrm{~nm}$-thick amorphous layer on the valley walls.

"Section II" and "section III" correspond to the nonintentionally irradiated regions of the structure. Section II is about $1.5 \mu \mathrm{m}$ large. It contains an amorphous layer (free of Ga nanoparticles) with a thickness of $35 \mathrm{~nm}$ close to the valley. While the distance from the valley increases, its thickness continuously decreases. At a distance of about $1 \mu \mathrm{m}$ from the valley, the amorphous layer starts to be sandwiched between the damaged crystalline superficial layer and the substrate. At a distance of $1.5 \mu \mathrm{m}$ from the valley, the amorphous layer completely collapses and transforms into a $25 \mathrm{~nm}$-thick damaged crystalline layer (see the strong contrast variation in the left part of left-side insert). Here, we define the onset of section III.

The overall analysis of this structure indicates that: (i) within the purposely irradiated area (section I), the Ga dose was largely sufficient to sputter and amorphize the whole $\mathrm{SiC}$ superficial layer and to induce Ga precipitation in the form of nanoparticles; (ii) at a distance of more than $1.5 \mu \mathrm{m}$ from this area (section III), the Ga dose was much smaller than in section I and the damage it induced has not reached a threshold value necessary for $\mathrm{SiC}$ amorphization; (iii) in between these sections, the Ga dose was lower than in section I but still high enough to induce complete amorphization of a $\mathrm{SiC}$ superficial layer close to section I. At a distance more than $1 \mu \mathrm{m}$ from the valley, the dose decreased down to a value sufficient only for a partial embedded amorphization of the superficial layer. In that case, the threshold damage value necessary for $\mathrm{SiC}$ amorphization could be reached only at the peak of the vertical damage profile, but not above or below. ${ }^{11}$

Figure 5(c) shows the ADF-STEM image of the structure irradiated with 10 times lower dose of Ga ions. As in the previous case, one can still distinguish sections I and II. Section I contains a $20 \mathrm{~nm}$-deep $\sim 1 \mu \mathrm{m}$ large valley which corresponds to the intentionally irradiated area. The $40 \mathrm{~nm}$-thick amorphous layer in the valley contains a band of Ga nanoparticles within its upper part (see the characteristic white band over the dark amorphous $\mathrm{SiC}$ ). The compositional nature of these clusters was confirmed by EELS-STEM analysis similar to that shown in Fig. 5(b). Section II still contains an amorphous layer (free of Ga nanoparticles) being twice thinner than that in the valley.

We have compared these data with that previously obtained for Si substrate irradiated by Ga ions with the same dose of $1 \times 10^{17} \mathrm{~cm}^{-2}$. Figure $5(\mathrm{~d})$ shows the Bright-field image of this structure. As for $\mathrm{SiC}$, the structure contains three different regions labelled as sections I, II, and III. The valley in section I contains an amorphous layer with (likely) $\mathrm{Ga}$ nanoparticles embedded inside. In contrast to the $\mathrm{SiC}$ structure, the nanoparticles are well spatially arranged in a double layer. The depth of the valley being of $36 \mathrm{~nm}$, the thickness of the sputtered Si layer is almost twice the thickness of the sputtered SiC layer. This finding is in good agreement with the predictions. ${ }^{30}$

Overall, SSRM and TEM analyses result in comparable radii for measureable effects and the electrical behaviour of the material can be explained combining both methods. The arrangement of $\mathrm{Ga}$ nanoclusters in the amorphous layer of 


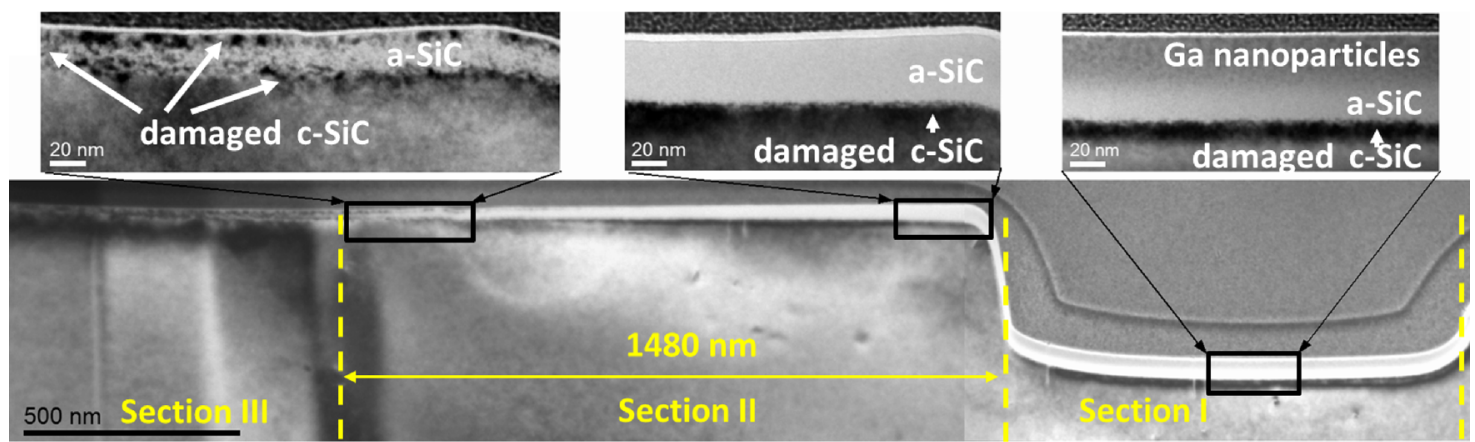

(a)
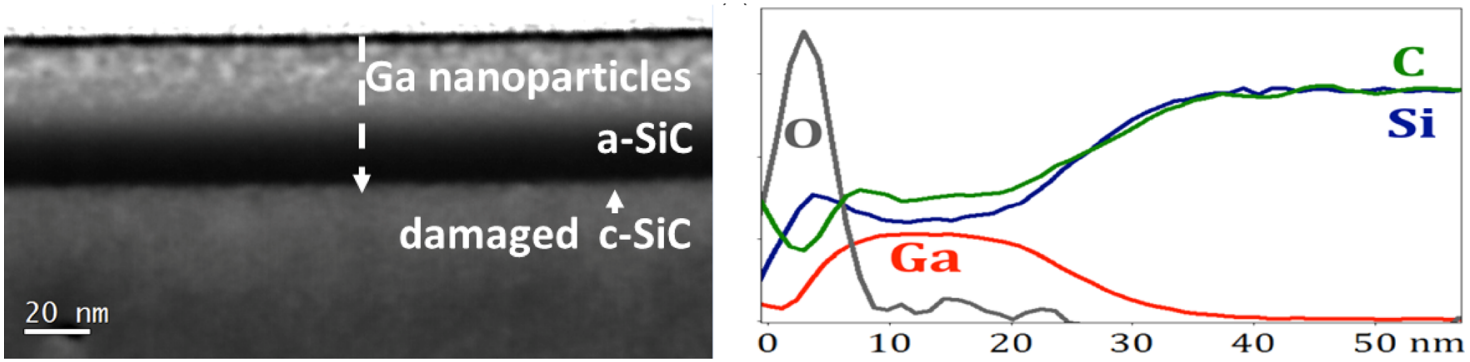

(b)

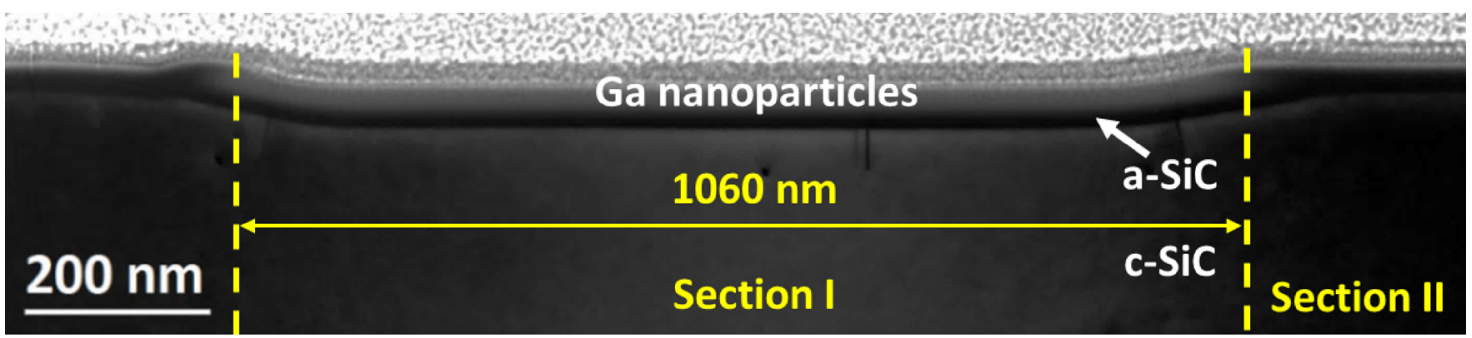

(c)

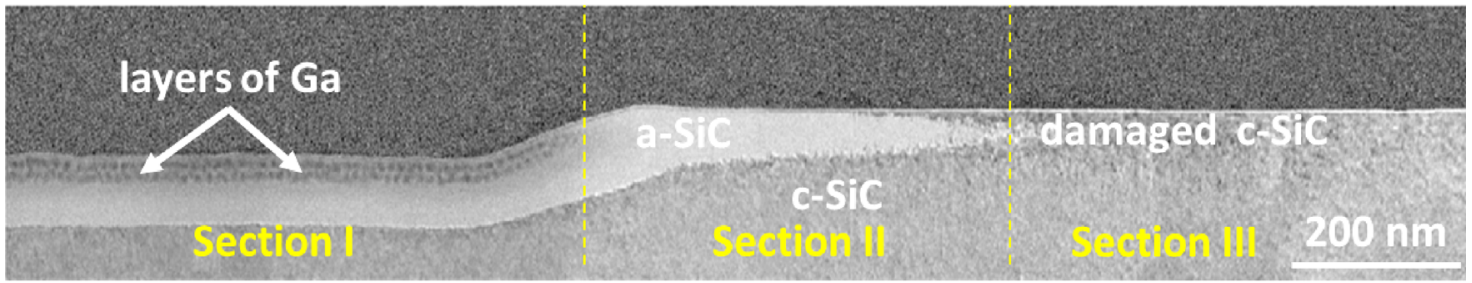

(d)

FIG. 5. (a) Bright-field TEM image of the $\mathrm{SiC}$ substrate irradiated with a Ga dose of $1 \times 10^{18} \mathrm{~cm}^{-2}$. Section I corresponds to the intentionally irradiated area while sections II and III to the non-intentionally irradiated areas. Enlarged views of the different sections are also shown. The dark spots in the right-side view correspond to Ga nanoparticles in the amorphous layer of the valley. The middle view shows an amorphous layer free of nanoparticles. The left-side view describes a transition from an embedded amorphous to damaged crystalline state in the superficial layer. (b) Annular Dark-Field (ADF) STEM image of the part of the valley in the $\mathrm{SiC}$ substrate irradiated by FIB with a Ga dose of $1 \times 10^{18} \mathrm{~cm}^{-2}$ and EELS-STEM elemental profiles acquired along the white dashed arrow evidencing the presence of Ga nanoparticles in the amorphous layer. (c) ADF-STEM image of the SiC substrate irradiated with a Ga dose of $1 \times 10^{17} \mathrm{~cm}^{-2}$. The brighter upper band in the dark amorphous SiC layer corresponds to a layer of Ga nanoparticles present in the valley (section I) but not in the outer regions (section II). (d) Bright-field image of a Si substrate irradiated with a Ga dose of $1 \times 10^{17} \mathrm{~cm}^{-2}$ [Reprinted with the permission from Rommel et al. J. Vac. Sci. Technol. B 28, 595 (2010). Copyright 2010, American Vacuum Society]. Likely Ga nanoclusters form the well-arranged double layer within the amorphous $\mathrm{Si}$, whereas they are randomly distributed within the amorphous $\mathrm{SiC}$ (c). a-SiC and c-SiC refer to amorphous $\mathrm{SiC}$ and crystalline $\mathrm{SiC}$, respectively and accordingly, a-Si and c-Si to amorphous and crystalline $\mathrm{Si}$.

the purposely irradiated area is similar to that observed for the lower dose $\left(1 \times 10^{17} \mathrm{~cm}^{-2}\right)$ and consequently, the measured spreading resistance is nearly identical [see red curve in Fig. 4(d)]. On the other side, the TEM analysis of the Si sample [Fig. 5(d)] shows that Ga nanoclusters within the irradiated valley are not randomly distributed but arranged in a well-organized precipitation layer. This layer effectively forms a conductive layer and, thus, increases the effective contact area of the AFM set-up significantly which leads to a further decrease in spreading resistance compared with $\mathrm{SiC}$ [see the difference of red and orange curves in Fig. 4(d)] for highest ion doses).

Overall, the two materials show very similar behaviour for FIB irradiation in the SSRM measurements for a large range of doses. $\mathrm{SiC}$ is in general slightly less sensitive toward FIB irradiation, most likely due to the lower intrinsic carrier 
lifetime of the material. In addition, the SSRM measurements as such are less sensitive due to the higher contact resistance for the wide bandgap material. Thus, for $\mathrm{SiC}$, smaller detectable affected areas and higher base resistances can be observed. The formation of a very distinct $\mathrm{Ga}$ precipitation layer in $\mathrm{Si}$ but not in $\mathrm{SiC}$ for high doses increases the difference between SSRM measurement results for the investigated structures on $\mathrm{SiC}$ and $\mathrm{Si}$ further, as the effective resistance drops significantly for $\mathrm{Si}$ at higher doses.

\section{B. Conductive atomic force microscopy}

c-AFM is applied to investigate the comparability of different electrical AFM methods and to gain a deeper insight into the current flow depending on sample position and applied voltage. To this end, the structure irradiated with a Ga dose of $1 \times 10^{17} \mathrm{~cm}^{-2}$ was mapped with c-AFM at $-3 \mathrm{~V}$ sample bias, see Fig. 6(a). The map is comparable to the SSRM measurement [Fig. 4(b)], showing the highly conductive section in the centre and the lower conductivity in the affected area surrounding the purposely irradiated area. In order to obtain very detailed data, 900 individual I-V curves were taken in an array of $30 \times 30$ measurements as indicated by the red dots in Fig. 6(a). The distance between single measurement points was set to $330 \mathrm{~nm}$ to avoid overlaps and dependences from one curve to another. The single curves were grouped together in a circular fashion to accumulate for the circular shape of the FIB beam and then averaged.

The four measurements in the centre of the affected area, i.e., the purposely irradiated area, were grouped and averaged as "circle 1". From there on outwards, each $500 \mathrm{~nm}$ another circle was drawn and the associated curves averaged and named after their specific circle. Measurements directly on the circumferences were discarded and not taken into account, to allow for distinguishable areas. The outermost area covered all curves exceeding the ten inner circles (equals 214 measurements) and was labelled "circle 11."

The measurements were taken in forward bias direction according to the Schottky contact measured in Fig. 3, thus from $0 \mathrm{~V}$ to $-5 \mathrm{~V}$. The voltage speed was set to $1 \mathrm{~V} / \mathrm{s}$, the current sensitivity to $100 \mathrm{nA} / \mathrm{V}$ and the number of measurement points for each curve amounted to 256. The curves were $\mathrm{V}$-averaged in accordance with the explanation in Sec. II. The plots are visualized in Fig. 6(b). The red graph shows the average of five I-V curves that were measured approx. $50 \mu \mathrm{m}$ away from the irradiated structure to ensure a measurement completely unaffected by the FIB irradiation. The green graph represents the first circle and has even higher current flow than the unaffected area, which corresponds to the observation based on the SSRM results. The second (magenta) and third (top black) graphs show almost no current flow even at bias voltages as high as $-5 \mathrm{~V}$. Again, this is in very good accordance with the high resistances measured by SSRM. From there on, the orange arrow [see Fig. 6(b)] indicates the results for the increasing number of circles up to circle 11 (blue). It is apparent that the current flow increases with the index of circles. This observation is also in good accordance with the SSRM measurements.

For a quantification of the results, the currents at $-3 \mathrm{~V}$ bias voltage were taken for all circles [indicated by the grey dashed line in Fig. 6(b)] and the according resistances were calculated (by simply dividing applied voltage and measured current). As the current within circle 1 already reaches the compliance value of $-450 \mathrm{nA}$ before the voltage of $-3 \mathrm{~V}$ was reached, the compliance value was taken as value for the resistance calculation (i.e., $-450 \mathrm{nA})$. The results are shown in Fig. 6(c) in comparison with the measurements of the SSRM and c-AFM mapping. The c-AFM matrix (blue curve) is plotted using the values from the calculation. For the SSRM and c-AFM sections (black and red curves, respectively), profiles of the corresponding maps through the centre of the irradiated area were taken. The sections have a width of $330 \mathrm{~nm}$ to allow averaging of the values and to obtain a better signal to noise ratio. Regarding the results in Fig. 6(c), the three different methods show very good comparability. The width of the different areas (sections I-III) is very similar and only small differences in the absolute values are visible at the centre and the

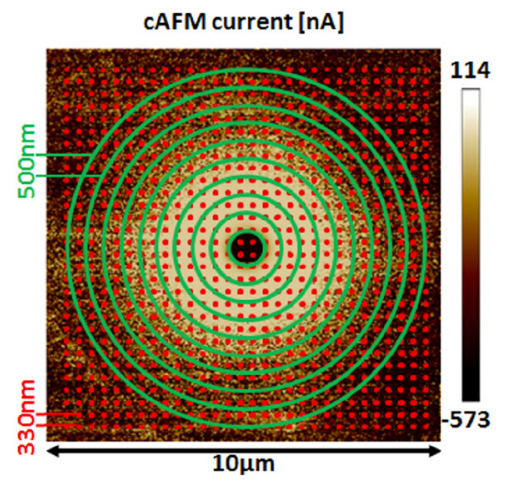

(a)

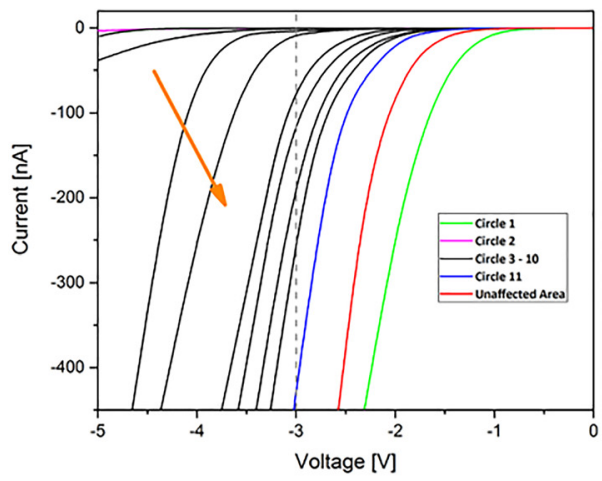

(b)

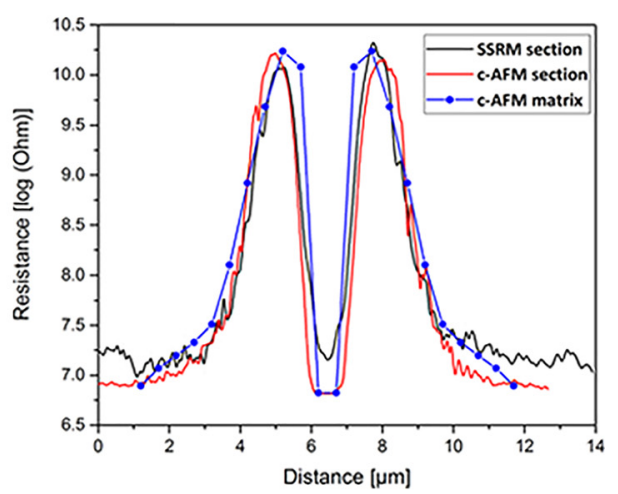

(c)

FIG. 6. (a) c-AFM current map measurement of the structure irradiated with a dose of $1 \times 10^{17} \mathrm{~cm}^{-2}$ at $-3 \mathrm{~V}$ sample bias. The red dots indicate the positions where I-V curves were taken, arranged in a $30 \times 30$ matrix. The ramps were measured from $0 \mathrm{~V}$ to $-5 \mathrm{~V}$ with a point distance of 330 nm to avoid overlap of the measurements and thus interactions with previously measured surfaces. The results were averaged over different radial areas, indicated by the green circles, numbered from 1 to 11 . V-averaged I-V curves from matrix (a) are shown in (b). The orange arrow indicates the graphs corresponding to the circles starting from circle two (magenta) to circle eleven (blue). The unaffected area was measured $50 \mu \mathrm{m}$ away from the irradiated structure to ensure no influence of Ga ions on the measurement and is an average of five curves. (c) Comparison of sections from SSRM and c-AFM map as well as c-AFM matrix measurements of the specified structure. 
edges of irradiation. Those differences are probably caused by the different amplifiers used for the electrical measurements and thus, different sensitivities at the limits of the measureable range.

The results prove that both methods (SSRM and c-AFM) can be used successfully to investigate the damage caused by FIB irradiation on SiC. As explained in chapter II, the samples were immediately measured after FIB irradiation to decrease the growth of native oxide and, thus, every sample was only measured once with one of the methods. Therefore, all measurements were performed on different individual samples and the achieved comparability proves that the size and effect of the FIB damage are quantitatively reproducible.

\section{CONCLUSIONS}

This work focused on the detailed investigation of FIB induced damage beyond purposely irradiated areas in the case of silicon carbide. For a better understanding and interpretation of the results, the experiments were set up comparably to those on silicon discussed in previous works. ${ }^{5,29}$ The high sensitivity of the electrical AFM, especially SSRM, for very low defect levels renders this method relevant for the quantification of any FIB induced damage which modifies the conductivity of the target material. It was proven that SSRM is still applicable despite the hardness of $\mathrm{SiC}$ and its large bandgap, and provides the option to compare $\mathrm{SiC}$ with other materials. It was shown that the lateral spread of damage behaves very similarly for silicon and silicon carbide substrates with comparable bulk resistances. The damaged sample region is mainly caused by the Gaussian shape of the center of the focused ion beam and its beam tail, which is basically independent of the substrate material. The small differences between the results for $\mathrm{SiC}$ (this work) and for $\mathrm{Si}^{5}$ outside of the purposely irradiated area are mainly explained by the different sensitivity toward the measurement set-up due to the contact resistance between AFM tip and the different substrates. The interpretation and explanation of the electrical results are supported and complemented by TEM analyses, allowing the correlation of three sub-regions with different electrical behaviours with different morphologies, i.e., amorphized regions with either very high amount of $\mathrm{Ga}$ or rather low $\mathrm{Ga}$ content and regions which show Ga-induced crystal defects.

Furthermore, the absolute resistance values within the purposely irradiated areas are comparable for $4 \mathrm{H}-\mathrm{SiC}$ and $\mathrm{Si}$ as long as the $\mathrm{Ga}^{+}$ion doses are below $3 \times 10^{16} \mathrm{~cm}^{-2}$. As proven by TEM, for higher doses, the formation of a distinct Ga precipitation layer in Si reduces the effective spreading resistance drastically, while the Ga ions remain randomly arranged in $\mathrm{SiC}$ without forming any kind of connected layer and thus, the resistance stays approximately constant.

Changes to the standard c-AFM measurement and analysis that improve the quality and reproducibility of c-AFM in general have been presented. First, the I-V curves are not ramped from minimum to maximum voltage in a single ramp, but rather split into two separate ramps with $0 \mathrm{~V}$ as common point. This drastically increased the reproducibility of the measurements as the voltage is increased steadily compared with the sudden voltage change in the standard set-up when measuring the ramp from minimum to maximum voltage or vice versa. Second, the averaging that is applied to the c-AFM data has to be adjusted according to the voltage regime. Usually, c-AFM curves are averaged using the standard approach of taking the average current at each voltage. However, it could be shown that for measurements in the forward bias regime, the averaging is more reliable and gives more realistic curves if instead the voltages are averaged for each current, whereas for the reverse bias regime, the common approach is preferable.

Additionally, the comparison of c-AFM ramp measurements and c-AFM as well as SSRM map measurements has shown that the lateral damage spread is not only constant between the measurement methods, but also constant over various samples and, thus, validates the results.

\section{ACKNOWLEDGMENTS}

The research leading to these results received funding from the European Union Seventh Framework Programme under Grant Agreement 312483 - ESTEEM2 (Integrated Infrastructure Initiative-I3).

${ }^{1}$ M. E. Schmidt, Z. Johari, R. Ismail, H. Mizuta, and H. M. H. Chong, Microelectron. Eng. 98, 313 (2012).

${ }^{2}$ J. Gierak, A. Madouri, A. L. Biance, E. Bourhis, G. Patriarche, C. Ulysse, D. Lucot, X. Lafosse, L. Auvray, L. Bruchhaus, and R. Jede, Microelectron. Eng. 84, 779 (2007).

${ }^{3}$ L. A. Giannuzzi, J. L. Drown, S. R. Brown, R. B. Irwin, and F. A. Stevie, Microsc. Res. Tech. 41, 285 (1998).

${ }^{4}$ K. D. Sattler, Handbook of Nanophysics: Principles and Methods (CRC Press, 2010).

${ }^{5}$ M. Rommel, G. Spoldi, V. Yanev, S. Beuer, B. Amon, J. Jambreck, S. Petersen, and A. J. Bauer, J. Vac. Sci. Technol. B 28, 595 (2010).

${ }^{6}$ W. Brezna, H. Wanzenböck, A. Lugstein, E. Bertagnolli, E. Gornik, and J. Smoliner, Semicond. Sci. Technol. 18, 195 (2003).

${ }^{7}$ M. F. Russo, Jr., M. Maazouz, L. A. Giannuzzi, C. Chandler, M. Utlaut, and B. J. Garrison, Appl. Surf. Sci. 255, 828 (2008).

${ }^{8}$ J. Huang, M. Loeffler, U. Muehle, W. Moeller, H. Mulders, L. Kwakman, and E. Zschech, Microsc. Microanal. 21, 1839 (2015).

${ }^{9}$ Z. Liao, T. Zhang, M. Gall, A. Dianat, R. Rosenkranz, R. Jordan, G. Cuniberti, and E. Zschech, Appl. Phys. Lett. 107, 013108 (2015).

${ }^{10}$ A. Lugstein, B. Basnar, G. Hobler, and E. Bertagnolli, J. Appl. Phys. 92, 4037 (2002).

${ }^{11}$ G. B. Assayag, C. Vieu, J. Gierak, P. Sudraud, and A. Corbin, J. Vac. Sci. Technol. B 11, 2420 (1993).

${ }^{12}$ Y. Drezner, Y. Greenzweig, S. Tan, R. H. Livengood, and A. Raveh, J. Vac. Sci. Technol. 35, 011801 (2017).

${ }^{13}$ T. Bever, G. Jäger-Waldau, M. Eckberg, E. T. Heyen, H. Lage, A. D. Wieck, and K. Ploog, J. Appl. Phys. 72, 1858 (1992).

${ }^{14}$ W. Brezna, H. Wanzenböck, A. Lugstein, E. Bertagnolli, E. Gornik, and J. Smoliner, Phys. E (Amsterdam) 19, 178 (2003).

${ }^{15}$ G. Spoldi, S. Beuer, M. Rommel, V. Yanev, A. J. Bauer, and H. Ryssel, Microelectron. Eng. 86, 548 (2009).

${ }^{16}$ B. Ozpineci, L. M. Tolbert, S. K. Islam, and M. Chinthavali, "Comparison of wide bandgap semiconductors for power applications," in Proceedings of the 10th European Conference on Power Electronics and Applications (2003).

${ }^{17}$ A. Agarwal and S. Haney, J. Electron. Mater. 37, 646 (2008).

${ }^{18}$ P. De Wolf, T. Clarysse, W. Vandervorst, J. Snauwaert, and L. Hellemans, J. Vac. Sci. Technol. B 14, 380 (1996).

${ }^{19}$ P. D. Wolf, T. Clarysse, W. Vandervorst, L. Hellemans, P. Niedermann, and W. Hänni, J. Vac. Sci. Technol. B 16, 355 (1998).

${ }^{20}$ P. De Wolf, E. Brazel, and A. Erickson, Mater. Sci. Semicond. Proc. 4, 71 (2001).

${ }^{21}$ M. Lanza, Conductive Atomic Force Microscopy (Wiley-VCH, 2017).

${ }^{22}$ G. Binning, C. F. Quate, and C. Gerber, Phys. Rev. Lett. 56, 930 (1986).

${ }^{23}$ Q. Zhong, D. Inniss, K. Kjoller, and V. B. Ellings, Surf. Sci. 290, L688 (1993). 
${ }^{24}$ M. Rommel, J. D. Jambreck, M. Lemberger, A. J. Bauer, L. Frey, K. Murakami, C. Richter, and P. Weinzierl, J. Vac. Sci. Technol. B 31, 01A108 (2013)

${ }^{25}$ D.-Z. Guo, S.-M. Hou, G.-M. Zhang, and Z.-Q. Xue, Appl. Surf. Sci. 252, 5149 (2006).

${ }^{26}$ K. Murakami, M. Rommel, V. Yanev, A. J. Bauer, and L. Frey, AIP Conf. Proc. 1395, 134 (2011).

${ }^{27}$ M. Rumler, M. Rommel, J. Erlekampf, M. Azizi, T. Geiger, A. J. Bauer, E. Meißner, and L. Frey, J. Appl. Phys. 112, 034909 (2012).

${ }^{28}$ R. Menzel, K. Gärtner, W. Wesch, and H. Hobert, J. Appl. Phys. 88, 5658 (2000).
${ }^{29}$ S. Dhar, R. P. Davis, and L. C. Feldman, Nanotechnology 17, 4514 (2006).

${ }^{30}$ S. K. P. Veerapandian, S. Beuer, M. Rumler, F. Stumpf, K. Thomas, L. Pillatsch, J. Michler, L. Frey, and M. Rommel, Nucl. Instrum. Methods Phys. Res., Sect. B 365, 44 (2015).

${ }^{31}$ S. Beuer, V. Yanev, M. Rommel, A. J. Bauer, and H. Ryssel, J. Phys.: Conf. Ser. 100, 052007 (2008).

${ }^{32}$ E. Platzgummer, A. Biedermann, H. Langfischer, S. Eder-Kapl, M. Kuemmel, S. Cernusca, H. Loeschner, C. Lehrer, L. Frey, A. Lugstein, and E. Bertagnolli, Microelectron. Eng. 83, 936 (2006).

${ }^{33}$ T. Kimoto and J. A. Cooper, Fundamentals of Silicon Carbide Technology (Wiley, 2014). 